\title{
Fromages de chèvre
}

\author{
par \\ le docteur Yves SAURAIS \\ Vétérinaire à Saint-Maixent-L'Ecole (79)
}

Faire un excellent fromage de chèvre est un petit tour de force en soi qui met en cuvre technique et empirisme : technique de la fabrication, empirisme de la finition et de la conservation.

A notre époque, la technique industrielle, préoccupée par une production sans cesse croissante, met en œuvre des moyens qui aboutissent à un produit de bonne qualité mais qui n'offrent plus au consommateur gastronome la joie de la découverte des merveilleux fromages de bonne femme du début de ce siècle.

A cette époque, quelques heures après la traite, on emprésurait le lait et on l'entreposait dans un local frais. Au bout de 2 ou 3 j, on faisait égoutter le caillé onctueux dans un linge fin et propre, puis on le moulait avant de le saler et de le transporter dans un local frais et humide pour qu'il s'affine.

Trois semaines plus tard, on allait le vendre au marché.

C'était beaucoup plus qu'une technique, un art de tradition orale où chacun s'ingéniait à mettre une touche personnelle.

La grande révolution dans le domaine de la fabrication a commencé en 1906 à Bougon (Deux-Sèvres) où fut créée la première fromagerie coopérative. Depuis cette époque, et particulièrement dans la région Poitou-Charentes, de nombreuses fromageries se sont installées, alors que disparaissait, petit-à-petit toute la production fermière.

La quantité de lait traité atteindra, en 1970, 85 millions d'hl pour la région, et, devant l'importance de cette production, la plupart des usines ont modernisé leurs moyens de fabrication, afin de faire face aux augmentations futures du volume collecté et d'améliorer sans cesse qualité, régularité et finesse du fromage.

En matière de production fromagère la qualité future ne s'obtient qu'en respectant toutes les phases du traitement du lait :

Récolte ; collecte ; conservation ; gélification ; égouttage du caillé ; moulage ; salage ; maturation ou affinage ; emballage ; expédition et finition dernière étape avant la dégustation. 


\section{LA RECOLTE}

Bien que destiné à une chaîne de fabrication industrielle, le lait provient en grande partie d'une forme d'élevage traditionnel, permettant, étant donné son prix ( $1 \mathrm{~F}$ le litre en moyenne), à des agriculteurs de survivre sur de petites superficies.

C'est dire que les conditions d'habitat ne sont pas toujours très satisfaisantes. La traite, le plus souvent, se fait manuellement. Il arrive même que la chèvre soit traite par derrière ! Il n'y a pas de local de traite. Par manque d'information ou par excès de zèle la désinfection des ustensiles se fait à l'aide de produits tels que l'ammonium quaternaire ou l'eau de Javel (corrosif pour les bidons). Le meilleur traitement et le moins coûteux à la ferme est encore l'eau bouillante.

Depuis quelques années, des unités de production importantes se sont développées. Elles ont opté, le plus souvent, pour la stabulation. Dotées d'un matériel de traite moderne elles produisent un lait de qualité.

De toute façon, cette recherche de la qualité est à l'ordre du jour et dans un proche avenir le lait sera payé pour sa qualité bactériologique. Le contrôle se fait par l'épreuve de réduction du bleu de méthylène ou de la résazurine.

On fait aussi des tests de fermentation pour déceler les microorganismes pouvant provoquer un caillé indésirable. Le lait doit être exempt de substances inhibitrices naturelles ou provenant de la croissance de bactéries productrices d'antibiotiques.

\section{CONSERVATION}

Collecté de ferme en ferme, le lait est acheminé en camion citerne jusqu'au quai. Là, refroidi à $8^{\circ} \mathrm{C}$, stocké dans des tanks, il subit un chauffage dont les températures d'action varient selon les fabrications ( $56^{\circ} \mathrm{C}$ en général).

Ce chauffage favorise la fermentation du lactose, tendant à sa transformation en acide lactique titré en degrés Dornic.

La fabrication d'un fromage comprend trois phases essentielles :

1) Formation du gel de caséine - coagulation ;

2) Déshydratation partielle de ce gel par synérèse, c'est-à-dire par contraction des micelles qui le forment. Egouttage du caillé ;

3) Maturation enzymatique du gel déshydraté. La pasteurisation ayant détruit une partie de la flore lactique, on réensemence le lait avec un levain.

Puis on emprésure par addition de présure à $1 / 50001$. 


\section{GELIFICATION}

La gélification procède donc de deux phases simultanées, une lactique, l'autre par la présure, aboutissant à la réunion collö̈dale des matières azotées : le caillé.

On observe au cours de la coagulation du lait par la présure, deux réactions successives. La première enzymatique qui transforme la caséine en para-caséine, l'autre correspondant à la phase de coagulation proprement dite et indépendante de la présure. Sa vitesse varie avec la température.

Le $\mathrm{pH}$ du lait intervenant aussi dans la rapidité du temps de coagulation ( $\mathrm{pH}<6$; temp. minimum), on conçoit alors que le fromager ait à surveiller et contrôler d'une part le $\mathrm{pH}$ d'autre part la température, car les formats de ses fabrications ainsi que leur qualité organoleptique sont fonction de ces variantes.

Technique de prise rapide. $26^{\circ} \mathrm{C}$ formats ronds plats, $8 \mathrm{cc} / 100 \mathrm{l}$.

Technique de prise lente. 23 C. Ste-Maure, Bûchette, Pyramides, Chabis, $4 \mathrm{cc} / 1001$.

Précisons que les doses d'emprésurage et d'ensemencement varient sensiblement avec la composition du lait, étant tributaires des conditions climatiques et des saisons, de l'alimentation plus ou moins équilibrée, de la nature des pâturages.

\section{EGOUTTAGE}

La conduite de l'égouttage du caillé requiert autant d'attention que de doigté.

Le caillé de chèvre est, nous l'avons vu, lactique et présure à la fois. Le caillé lactique en effet est cassant, friable et poreux et son égouttage s'accomplit presque seul, l'élimination du sérum se fait très lentement, la contraction des micelles, ou synérèse, étant relativement faible.

Le caillé présure est au contraire mou, gélatineux, presque imperméable. Cette imperméabilité est due à la polymérisation de ses micelles par le calcium. L'égouttage de ce caillé ne peut s'accomplir spontanément qu'après acidification et reprise du caractère lactique (dépolymérisation). Il est nécessaire d'intervenir avant cette phase par un travail approprié du caillé (tranchage).

L'égouttage est donc dépendant de la nature du caillé, plus ou moins lactique ou présure.

Egouttage et coagulation sont deux phases complémentaires et concourant à régler à un point critique optimum les caractères physico-chimiques du fromage égoutté en vue de sa maturation. 
Un caillé lactique égoutté spontanément donne une pâte de grande plasticité sans la moindre cohésion : pâtes fraîches.

Un caillé présure égoutté mécaniquement avant acidification donne un fromage à pâte ferme plus ou moins élastique de très grande cohésion : Cantal, Giuyère.

Entre ces deux natures de caillé on peut en utilisant les deux phases parallèlement obtenir toutes les textures de pâtes.

Signalons encore qu'un caillé présure est fortement minéralisé en calcium, le lactique étant déminéralisé.

Le gel est obtenu $15 \mathrm{~h}$ après l'emprésurage pour les prises lentes et $2 \mathrm{~h} 30$ pour les prises rapides.

Le caillé est alors aussitôt mis en forme.

\section{MOULAGE}

Le moulage traditionnel se fait en répartissant le caillé à la louche dans des moules perforés, sans fond, alignés sur des tables canelées dont l'inclinaison conditionne l'écoulement du sérum vers une fosse de réception. Au cours de cet égouttage qui se prolonge $18 \mathrm{~h}$ pour les prises rapides et $24 \mathrm{~h}$ pour les prises lentes, les moules sont retournés et le caillé égalisé.

Le moulage mécanique permet l'égouttage du caillé en masse dans des filtres de toile nylon animés d'un mouvement d'oscillation pendant $18 \mathrm{~h}$ environ. La pâte est passée en mouleuses pour l'obtention du fromage en continu. Ce procédé nouveau est utilisé pour la fabrication des fromages de formats cylindriques et des cubes de caillé destinés à la congélation.

Remarquons que la texture des caillés est différente selon qu'il s'agit de moulage traditionnel ou de moulage mécanique. La pâte traditionnelle possède un grain brut alors que la pâte mécanique a subi comme un pétrissage qui impose une conduite de l'affinage toute différente.

\section{SALAGE}

Les fromages démoulés sont répartis sur des claies, et le salage s'effectue par pulvérisation de saumure - le salage à la main a disparu. Cette opération très importante est facteur de la qualité gustative des futurs fromages. Elle s'accompagne d'une pulvérisation de penicillium qui stimule le démarrage de la flore superficielle.

Après un stage de $24 \mathrm{~h}$ en salle climatisée à $13^{\circ} \mathrm{C}$ les fromages sont retournés et, ensuite, dirigés vers les haloirs où ils vont subir la maturation enzymatique due à leurs ferments. 


\section{MATURATION}

Pendant $12 \mathrm{j}$, à $11^{\circ} \mathrm{C}$ et par 95 p. 100 d'hygrométrie le caillé va être le siège de transformations chimiques qui vont développer son arôme - goût épicé ou goût piquant, odeur de bouc, odeur ammoniacale, autant de qualités qu'il faut rechercher ou éviter.

Cette maturation est menée sous l'action de divers facteurs :

- enzymes naturelles du lait,

- présure,

- enzymes microbiennes provenant de la lyse des micro-organismes détruits lors des opérations précédentes,

- action simultanée s'exerçant de l'extérieur vers l'intérieur des cultures de penicillium pulvérisées sur la surface.

Le rôle des enzymes du lait est maintenant contesté sachant qu'elles ont un optimum d'activité à $\mathrm{pH} 8$ alors que les fabrications ont un $\mathrm{pH}$ de 5 à 5,5 .

La présure intervient dans le développement de l'arôme surtout en tant qu'agent métabolique. Elle forme des produits de dégradation, substances azotées, qui sont eux-mêmes repris par d'autres agents pour l'ultime conversion en produits responsables de l'arôme.

Caséine $+\mathrm{H}_{2} \mathrm{O} \rightarrow$ Protéose $+\mathrm{H}_{2} \mathrm{O} \rightarrow$ Peptones $+\mathrm{H}_{2} \mathrm{O} \rightarrow$ Peptides $+\mathrm{H}_{2} \mathrm{O}$ insoluble soluble ac. aminés.

Les acides aminés solubles sont considérés comme favorables au point de vue goût des fromages, à l'inverse des peptones dont le goût amer est nettement défavorable.

Les micro-organismes jouent de loin le rôle le plus important. Ils sont environ 500 millions/ml dont le système enzymatique, libéré après leur mort, va attaquer les molécules de peptones ou de lipides.

Parmi eux, les streptocoques lactiques (lactis et cremoris, homofermentaires acidifiants, diacetylactis, hétérofermentaire, aromatisant), les lactobacilles et les entérocoques.

Les protéinases et peptidases transforment les molécules de peptides en acides aminés que les décarboxylases provenant du système enzymatique des lactobacilles, coliformes et clostridies transforment à leur tour en amines et en $\mathrm{CO}_{2}$ selon le tableau ci-dessous :

Acide glutamique
Tyrosine
Lysine
Arginine
Histidine
Tryptophane

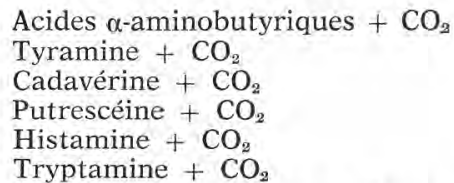

(Thèse de Doctorat de G. Silverman, Université de Cornell)

Les lipases microbiennes, issues des corps cellulaires des lactobacilles sont à l'origine des transformations de la matière grasse en 
acide butyrique libre et de la totalité des acides caproïque, caprylique et caprique.

Les penicillium, au cours de cette maturation, prolifèrent et produisent un feutrage serré et fin accompagné d'une légère pointe d'Ö̈dium.

L'activité du penicillium lui permet de lutter contre les germes indésirables et son rôle essentiel consiste à consommer l'acide lactique et le lactose en favorisant la neutralisation du caillé donc l'affinage.

\section{QUELQUES MALADIES DES PATES}

En fromagerie de chèvre, on utilise le $P$. candidum. La variété glaucum dont une espèce, le $P$. roqueforti, est utilisée dans les fabrications des bleus, communique le goût piquant qui les caractérise en saponifiant la matière grasse au cours de l'affinage. C'est principalement à lui que les fromages fermiers doivent leur feutrage bleuvert très particulier. Il existe en effet à l'état naturel. Il apprécie l'atmosphère assez confinée des caves et celliers. Il n'est pas surprenant de le trouver à la surface des fromages à moisissures superficielles, c'est la maladie du bleu. D'autres maladies du fromage sont dues à la présence de moisissures ou de bactéries étrangères présentes dans les haloirs. Citons rapidement : les aerobacter aerogenes provoquant le gonflement du caillé, le geotrichum candidum, agent responsable de la grosse peau et dont le développement est favorisé par une température exagérée des salles de moulage et de salage, le Mucor qui, en atmosphère confinée et humide, provoque le " poil de chat ».

\section{LES REPORTS}

La production du lait étant saisonnière, il se pose un problème de planification de la vente à la consommation et de normalisation de la fabrication. 75 p. 100 de la production est ramassée entre les mois d'avril et septembre, 25 p. 100 pendant la période d'octobre à mars. Il se pose donc un problème de conservation des laits qui n'est guère envisageable en l'état, étant donné le volume considérable à stocker. Conserver sous forme de poudre ou de lait concentré n'est pas non plus très valable, les résultats n'étant pas satisfaisants en ce qui concerne les caractères organoleptiques du fromage.

Le procédé en cours est celui de la conservation du caillé congelé en tunnel à $-40^{\circ} \mathrm{C}$. Le stockage s'effectue en chambre froide à $-22^{\circ} \mathrm{C}$ pendant 7 à 8 mois. Il faut reconnaître que des défauts de goût peuvent apparaître après conservation, défauts pratiquement inconnus dans les fromages préparés avec du caillé frais.

Des défauts d'oxydation peuvent se produire et le salage avant conservation accélère l'oxydation de la matière grasse. Les examens organoleptiques ont montré que les fromages préparés à partir de 
caillé congelé ne possédaient pas la même richesse de bouquet que les témoins. Il y aurait des pertes appréciables de certains acides cétoniques, de composés carbonylés à fonction aldéhyde.

Des recherches très poussées sont en cours pour essayer de pallier ces inconvénients car le procédé est avant tout d'ordre économique.

Pour la seule région Poitou-Charentes 1300 à 1500 t de caillé sont stockées congelées, soit près du $1 / 4$ de la quantité de fromages de chèvre produits dans la région.

\section{EMBALLAGE - EXPEDITION}

A la sortie du haloir, les fromages sont prêts à être livrés à la consommation. Si le papier sulfurisé demeure la matière idéalement poreuse, la cellophane présente une imperméabilité néfaste que l'on corrige par des perforations discrètes. Ce sont cependant les deux seuls emballages papiers reconnus utilisables pour les produits fromagers qui respirent et qui doivent poursuivre leur maturation à cœur.

L'expédition se fait en boîtes en bois munies de tasseaux permettant une aération optimum.

\section{FINITION}

Il ne reste plus au revendeur, qu'à livrer au consommateur un produit à point. Ce dernier tour de main n'est hélas l'apanage que de quelques trop rares maîtres affineurs, qui savent procéder à la finition idéale qui séduira le client.

Ce goût du public est en lui-même extrêmement variable et si les Parisiens aiment un fromage jeune, non piquant, à peine affiné, les gens de la région Rhône-Alpes, ou du Sud-Ouest le préfère plus corsé et un peu plus acide.

C'est de toute évidence au consommateur d'imposer en dernier ressort, les normes de qualité du fromage de chèvre qu'il déguste.

\section{REGLEMENTATION}

\section{A) Mentions obligatoires}

Les mentions obligatoires suivantes doivent être portées sur les étiquettes :

1) Dénomination du fromage.

2) Nom et adresse du fabricant, cette mention pouvant être remplacée par l'indication d'un numéro d'immatriculation délivré par le Préfet du département dans lequel est située la fromagerie. 
3) Lieu de fabrication (département ou région).

4) Indication de la teneur en matière grasse minimum pour $100 \mathrm{~g}$ de fromage ramené à l'état sec.

Les fromages non définis, fabriqués dans les exploitations agricoles, sont dispensés de l'étiquetage, mais seulement s'ils sont vendus par le producteur lui-même.

\section{B) Dénomination}

En plus des inscriptions obligatoires pour chaque fromage, les fromages :

- de chèvre (fabriqué exclusivement au lait de chèvre) porteront la dénomination "Fromage de chèvre",

- mi-chèvre (contenant au moins 25 p. 100 de lait de chèvre) porteront la dénomination "Fromage mi-chèvre », la bande jaune portant la mention : 75 p. 100 de lait de vache.

Par lettre circulaire du 8 juin 1966, il a été admis par l'Administration que la mention " $\mathrm{X} \mathrm{p} .100$ de matière grasse " pouvait être utilisée pour indiquer la teneur en matière grasse des fromages de chèvre de petit format, à condition que cette mention soit portée en caractères de dimension au moins égale au $2 / 3$ des caractères de la dénomination, et de la même apparence typographique.

\section{C) Apposition de la bande de couleur bleu foncé sur les étiquettes des fromages (circulaire $\mathrm{n}^{\circ} 166$ du 5 août 1954)}

Les fromages rappelant par leur forme ou leur aspect ceux définis, ainsi que tous autres fromages non définis d'une teneur en matière grasse inférieure à 40 p. 100 ne pourront être détenus ou transportés en vue de la vente, mis en vente ou vendus entiers, que munis d'une bande de couleur bleu foncé, apparente, portant en caractères blancs, d'au moins un centimètre de hauteur, l'indication de leur teneur en matière grasse par la formule : "X. g p. 100 de matière grasse ».

Cette bande devra avoir des dimensions suffisantes pour ne pas donner l'impression qu'elle est placée uniquement pour faire ressortir les lettres blanches qui y sont inscrites, c'est-à-dire que l'indication de la teneur en matière grasse ne devra pas couvrir entièrement la longueur de la bande. Elle devra être apposée sur chaque fromage.

\section{Remerciements}

Nos remerciements s'adressent tout spécialement $M$. Moineau, Directeur de l'Association Centrale des Laiteries Charentes-Poitou - 17 Surgères et M. Dupuis, Directeur de la Fromagerie de Soignon - 79 Saint-Maixentl'Ecole pour leur collaboration et l'aide technique qu'ils nous ont apportée. 